\title{
El riesgo operacional en las entidades financieras: una aproximación empírica a las cajas de ahorro españolas
}

\section{Operational Risk in Financial Institutions. An empirical approach to spanish saving banks}

\author{
Ana Fernández Laviada. Universidad de Cantabria \\ Francisco Javier Martínez García. Universidad de Cantabria
}

\begin{abstract}
RESUMEN El riesgo operacional no es nuevo. Sin embargo, el creciente interés y preacupación por su gestión y control, en lo que las entidades financieras sin duda alguna están a la vanguardia, sí es un fenómeno reciente. Además, el hecho de que el Nuevo Acuerdo de Capitales de Basilea lo haya incluido entre sus requerimientos de capital, aportando una definición del mismo para el sector, ha propiciado que las entidades empiecen a desarrollar un modelo completo de gestión que les permita su identificación, valoración, seguimiento, control y mitigación. En este contexto, el objetivo de nuestra investigación es analizar, de forma empírica, el grado de conocimiento y desarrollo alcanzado, así como las tendencias futuras en cuanto a la gestión del riesgo operacional en las entidades financieras españolas, centrándonos en este artículo en la situacion que presenta el subsector de las Cajas de Ahorros.
\end{abstract}

PAIABRAS CLAVE Riesgo operacionail; Cajas de Ahorros; Entidades Financieras; Gestion de Riesgos.

\begin{abstract}
Operational risk (Opr), inherent in all business, is not new itself. However, the increasing interest about its management and control is something new for entities, and financial ones are the most advanced in this field. With the New Basel Capital Accord (known as Basel in) introducing, for the first time, explicit capital requirements for Opr and providing a definition of Opr, entities are beginning to develop a complete management framework that allows them to identify, asses, measure and later on control and mitigate it. In this context, the ain of our research is to empirically analyse the degree of knowledge and development as well as the trends for the future about Opr in Spanish Financial institutions. This paper focuses in particular on the state of the art in the savings bank sector.
\end{abstract}

KEY WORDS Operational risk; Savings banks; Financial institutions; Risk Management.

\section{INTRODUCCIÓN}

La desregulación, la globalización de los mercados, el aumento de la competencia, el estrechamiento de los márgenes, la creciente complejidad de los productos y la infiuencia de las nuevas tecnologías han sido, entre otros, los principales detonantes del aumento del riesgo en el conjunto de la economía. Las enticlades financieras, en particular; al tener como mexcancía objeto de su negocio el dinero, se encuentran inmersas dentro del denominado «concepto global del riesgo», por lo que su gestión se ha convertido en pieza clave no sólo de su éxito sino de su supervivencia.

La gestión del riesgo, en un sentido amplio, implica su identificación, evaluación, seguimiento o supervisión y control y debe proyectarse a todos los niveles organizativos de la entidad, desde una perspectiva diferente a la que hasta ahora se ha tenido. Los errores en las 
estimaciones sobre los posibles riesgos futuros pueden tener graves consecuencias no siempre subsanables. Bl mero control «a posteriori» resulta ya insuficiente y, junto a una clara concienciación sobre su importancia, ha de crearse un entorno que favorezca su gestión de manerd eficaz y eficienie.

Después de cuantiosos esfuerzos económicos y un largo proceso de avances en la medición y control de los riesgos de mercado, de crédito y de liqquidez, las entidades financieras comienzan a replantearse la situación, ya que a pesar de ello, una oporativa no autorizada, un error humano o un fallo en un proceso o sistema pueden poner en peligro a toda la organización, llevándola incluso a la quiebra, como ocurrió con la Banca Barings en 1995.

En los últimos años, las enormes pérdidas sufridas en múltiples entidades por fallos operacionales [Chorafas, 2001: 96-108; King, 2001: 21-34, y Hoffman, 2002: xxii-xxx], y sus graves consecuencias, han alertado a reguladores, normalizadores, supervisores y a las propias entidades sobre la necesidad y urgencia de gestionar el riesgo operacional, del mismo modo en que se han tratado y se tratan otros riesgos. El riesgo operacional, implícito en toda actividad, no es nuevo, pero sí lo es su gestión global e integrada en el sistema de gestión de riesgos de las entidades. Sin lugar a dudas, por su naturaleza es el riesgo más complejo de controlar y las entidades que no lo consigan pagarán sus consecuencias, a la vez que serán penalizadas a través del consumo de recursos propios [BASEL, 2003b].

Para empezar, indicar que en los últimos años se ha producido una progresiva evolución hacia una definición de riesgo operacional de aceptación general, propuesta y defendida por The Basel Committee on Banking Supervision, en adelante, Comité de Basilea, que lo identifica como: el riesgo de pérdidas resultantes de procesos internos inadecuados o defectuosos, personal, sistemas o como resultado de acontecimientos externos [BASEL, 2003aj. El mayor conflicto se ha generado por la confusión producida al identificarse con uno de sus componentes más importantes, el riesgo operativo, debido exclusivamente a las pérdidas por fallos en las operaciones como ya señalaban Hoffiman y Johnson [1996: 60-63] y Cade [1999].

No obstante, además del esfuerzo por llegar a una definición que delimite claramente lo que implica y abarca, resulta transcendental aceptar que debe tener un marco de gestión adecuado que necesariamente concrete el nivel de riesgo que desea asumir cada entidad, el modo en que será transferida la parte no deseada, así como los medios para poder identificar, evaluar, supervisar y controlar o mitigar este riesgo IFroot et al., 1994; Lam y Cameron, 1998; Aerts, 2001; Kennett, 2001; King, 2001, y FSA, 2003].

Es imprescindible establecer un modelo de estructura organizativa adecuado a la nueva situación de las entidades, que incluya las responsabilidades del Consejo y de la alta dirección, las funciones y responsabilidades del resto de las partes implicadas en el proceso, y los procedimientos más adecuados del gobierno de las entidades.

En los últimos años, y también fruto de los estrepitosos fracasos financieros [Hussain, 2000: 7-21], el debate, a nivel mundial, se ha centrado en el papel del Consejo y de la alta dirección, principales responsables de la actividad empresarial y de su control. Entre las buenas prácticas para el «buen gobierno de las sociedades» deberán estar incluidas, tanto la definición de las estrategias y políticas que garanticen una adecuada gestión global del riesgo, como el establecimiento, mantenimiento y supervisión de un buen sistema 
cer en la entidad un entorno cultural apropiado que facilite una efectiva gestión del riesgo operacional.

Una vez identificados los elementos que componen este riesgo operacional, y establecido el marco de gestión más apropiado a sus características, es paso obligado hablar de su control y mitigación, para lo cual el control y la auditoría internos juegan un papel fundamental [Dowd, 1998: 192-193, y Jan, 2001: 43-45]. Al menos por ahora, puesto que el Nuevo Acuerdo de Capital de Basilea limita su papel a la supervisión, con el fin de evitar posibles conflictos de intereses, $y$ hasta que se consiga un nivel de conocimiento y avance similar al de otros riesgos, en especial de mercado y de crédito, es el departamento de auditoria interna el primer impulsor de estas iniciativas.

La implicación responsable del Consejo y de la alta dirección, juntamente con la supervisiôn de la auditoría interna y la tutela de los suppervisores, deben garanízar l̇a cultura del «riesgo razonable» de las entidades financieras.

El sector financiero es el más avanzado en el tratamiento del riesgo operacional [Jameson, 1998: 139-160; Hussain, 2000; Jan, 2001, y Frost et al. 20011. Sus características y las nuevas exigencias de asignación de capital impuestas por el Comité de Basilea, favorecen este hecho. Su gran dependencia de la tecnología, su exposición a los riesgos asociados a transacciones cada vez más complejas, los enormes fiujos de datos que mueven los sistemas, muchos de los cuales también son vitales para el buen funcionamiento de las operaciones, junto a la creciente presión de los legisladores y supervisores, por garantizar İa continuidad de la actividad y la estabilidad del sistema, han hecho que el sector financiero tome paulatinamente conciencia de este riesgo.

Por todo ello, el objetivo principal que perseguimos con este trabajo es constatar empíricamente y evaluar la situación respecto al trataniento del riesgo operacional en las entidades financieras españolas, particularmente en las Cajas de Ahorros, comprobando la necesidad de profundizar en su análisis debido a la escasa atención que hasta ahora ha recibido desde todos los ámbitos, lo que a nuestro juicio, justifica el interés y la oportunidad de estudios como el que hemos realizado y presentamos a continuación.

\section{ESTADO DE LA CUESTIÓN: INVESTIGACIÓN PREVIA SOBRE EL RIESGO OPERACIONAL}

Señalaba Anthony [1990: 246] que la evidencia proporcionada por la práctica sobre la función de control de gestión procedía de alguna de las seis fuentes siguientes, a İas que clasificaba en orden descendente por su probable validez y a las que consideraba habría que añadir la experiencia personal: experimentos, observaciones, análisis estadísticos, encuestas, estudio de casos y modelos. En el ámbito de nuestra investigación, sostenía Tufano [1996: 1097] que los académicos sabemos muy poco sobre las prácticas de gestión de riesgos que siguen las entidades, a pesar de que cada vez éstas recurren más a nuevas técnicas y mecanismos para gestionar los riesgos a los que se enfrentan. Las entidades revelan muy pocos datos y aportan escasos detalles sobre sus políticas de riesgos, por lo que la mayoria de los estudios realizados se apoyan en la cuarta fuente identificada por Anthony, es decir, en los resultados de entrevistas o encuestas (surveys) y otros datos complementarios. 
A esta realidad debemos añadir las características del sector objeto de nuestro trabajo. El sistema financiero a nivel mundial se basa fundamentalmente en la confianza del público y se ha caracterizado siempre por su gran cautela y discreción, aunque también podría definirse como hermetismo, a la hora de publicar cualquier tipo de iniormación que pueda dañar o afectar de algún modo a esa confianza. Esto hace que apenas se pueda acceder a datos sobre fraudes, internos o externos, o sobre prácticas no autorizadas que hayan podido sufrir las entidades, y especialmente a tener noticias de las pérdidas que por uno $\mathrm{u}$ otro motivo pudieran haber tenido. Tan sólo līegamos a conocer una mínima parte cuando, fiuto de estas pérdidas, la entidad no tiene otra alternativa qque reconocer las situaciones que ya son totalmente insostenibles, como fieron los casos Barings, Daiwa, y otros mlcho más cercanos a nosotros, como el de Caja Granada.

Por todo ello, la literatura y los trabajos publicados en este campo son escasos. Sin embargo, una de las causas que ha favorecido la rápida evolución y el interés creciente por el riesgo operacional ha sido, precisamente, el esfuerzo realizado por diferentes autoridades reguladoxas en el ámbito internacional, como el Conité de Basilea o The Financial Services Authority (FSA), asociaciones, como The Futures and Options Association (FOA) 0 The In" ternational Swaps and Derivatives Association (ISDA), consultoras y otros organismos independientes. Así desde 1998 venimos asistiendo a la aparición de diferentes documentos, recomendaciones, trabajos y estudios que centran su atención en el riesgo operacional y que recogemos en la Tabla 1.

Tabla 1

DOCUMENTOS MÁS REIEVANTES SOBRE EL RZESGO OPERACTONAL

\begin{tabular}{|c|c|c|}
\hline OAGAUASMOS EMHSORES & DodumENTO & FECHA \\
\hline \multirow{8}{*}{$\begin{array}{l}\text { The Basel Committee } \\
\text { on Banking Supervision } \\
\text { (BASEL) }\end{array}$} & Operational risk management & Septiembre 1998 \\
\hline & Other risks. Discussion paper & Revisado en Abril 2000 \\
\hline & $\begin{array}{l}\text { Operational Risk. Supporting document to the New Basel } \\
\text { Capital Accord }\end{array}$ & Enero 2001 \\
\hline & $\begin{array}{l}\text { Working paper on the regulatory treatment } \\
\text { of operational risk }\end{array}$ & Septiembre 2001 \\
\hline & \multirow{3}{*}{$\begin{array}{l}\text { Sound practices for the management and supervision } \\
\text { of operational risk }\end{array}$} & Diciembre 2001 \\
\hline & & Agosto 2002 \\
\hline & & Febrero 2003 \\
\hline & New Basel Capital Accord (CP3) & Abril 2003 (*) \\
\hline \multirow{5}{*}{$\begin{array}{l}\text { The Financial Services Au- } \\
\text { thority (FSA) }\end{array}$} & Allocating regulatory capital for operational risk & 1999 \\
\hline & Senior management arrangements, systems and controls & 1399 \\
\hline & Operational risk systems and controls & 2002 \\
\hline & $\begin{array}{l}\text { Building a framework for operational risk management: } \\
\text { the FSA's observations }\end{array}$ & 2003 \\
\hline & Implementation of the capital accord for operational risk & 2003 \\
\hline \multirow{2}{*}{$\begin{array}{l}\text { The Futures and Options } \\
\text { Association (FOA) }\end{array}$} & $\begin{array}{l}\text { A review of regulatory capital requirements for EU credit } \\
\text { institutions and investment firms. Consultation } \\
\text { Document }\end{array}$ & 2000 \\
\hline & $\begin{array}{l}\text { Operational Risk. The Viability of an insurance/Capital } \\
\text { Market Solution }\end{array}$ & \\
\hline
\end{tabular}


TABLA 1

DoCUMENTOS MÁs RELEVANTES SOBRE EL RJESGO OPERACIONAL (CONt.)

\begin{tabular}{|l|l|c|}
\hline \multicolumn{1}{|c|}{ ORGAHSMOS EMHSORES } & \multicolumn{1}{|c|}{ DoCUMENTO } & FECHA \\
\hline $\begin{array}{l}\text { The Canadian } \\
\text { Treasurer (TCT) }\end{array}$ & The final frontier: managing operational risk & 1998 \\
\hline $\begin{array}{l}\text { The International Swaps } \\
\text { and Derivatives Association } \\
\text { (ISDA) }\end{array}$ & Operational risk regulatory approach. Discussion paper & 2000 \\
\hline $\begin{array}{l}\text { Office of the Comptroller } \\
\text { of the Currency (OCC) }\end{array}$ & $\begin{array}{l}\text { Supervisory guidance on operational risk advanced } \\
\text { measurement approaches for } \\
\text { regutatory capital }\end{array}$ & 2003 \\
\hline
\end{tabular}

FuEnt: Elaboración propia.

(*) El texto cefinitivo fue publicado el 26 de junio de 2004 con el título eInternational Convergence of Capital Measurement and Capital Standards: a Revised Framework"y traducide al espan̈ol «Convergencia Internacianal de medidas y normas de capital. Marco revisadow aunque cominnmente se le conoce como Basilea II.

El primero [BASEL, 1998], que publicó los resultados de un estudio realizado por el Comité con treinta de los mayores bancos del mundo, inició una serie de trabajos cuyo objetivo principal era conocer el estado de la cuestión en relación a la gestión del riesgo operacional en las mayores instituciones del mundo, avanzando así en la disciplina, y cuyo objetivo secundario era alcanzar un consenso sobre su definición. En ese documento, el Comité de Basilea declaraba y reconocía que gestionar el niesgo operacional estaba empezando a ser un importante rasgo distintivo de una buena gestión de riesgos en los mercados financieros modernos y animaba a los bancos a que compartieran con sus supervisores el desarrollo de nuevas técnicas para identifficar, evaluar, supervisar y controlar el riesgo operacional.

Durante 1998 y 1999, tres organizaciones: The International Swaps and Derivatives Association ${ }^{(3)}$ (ISDA), The British Bankers' Association (BBA) y Robert Morris Associates (RMA) patrocinaron un estudio sobre la gestión del riesgo operacional. Estas tres asociaciones, junto a los servicios de la multinacional PricewaterhouseCoopers (PwC), llevaron a cabo un trabajo que culminó, en diciembre de 1999, con el informe titulado «Operational risk: the next frontier», en el que se analizaba, amplia y detalladamente, la situación del riesgo operacional en cincuenta y cinco instituciones financieras de ámbito mundial.

Pero sin lugar a dudas, lo más decisivo ha sido que el Nuevo Acuerdo de Capital de Basilea incorpore a este riesgo en la misma categoría que lo hicieran previamente el riesgo de crédito o el de mercado. Ya en junio de 1999, en el documento consultivo elaborado por el Comité de Basilea, que revisaba el primer Acuerdo de Capital de 1988, se proponía que el capital regulatorio debía ser adecuado específicamente para cubrir «otros riesgos». Con ello trataba de reflejar el interés de hacer un Acuerdo más completo y sensible al riesgo, y al mismo tiempo reconocía la existencia e importancia de otros riesgos, aparte del de crédito y de mercado, ofreciendo incentivos para mejorar su gestión.

Por otra parte, The Institute of International Finance (IIF), grupo privado de investigación y apoyo que ofrece sus servicios a grandes bancos internacionales, creo entre 1999 y 2000, The Working Group on Operational Risk (WGOR) y The Industry Technical Working Group

(1) Creada en 1985 con el nombre de International Swap and Dealers Association, puede consultarse a travės de su página web: nttp:// www.isda.org todo lo relativo a esta activióad. 
on Operational Risk (ITWCOR), ambos con el objetivo fundamental de responder al referido documento consultivo del Comité de Basilea de junio de 1999. El ITWGOR es un órgano independiente, aunque a veces funciona como un subcomite del WGOR y del IHF, y los tres han trabajado en la pubìicación de documentos sobre el riesgo operacional. Finalmente en octubre de 2000 el ITWGOR adelantaba una definición de riesgo operacional utilizada por el IIF y que fue tomada como referente a partir de ese momento por el resto del sector financiero.

Por último, en nuestro país, resulta de especial interés referimos al trabajo llevado a cabo por una división de PwC [2002], especializada en la gestión giobal del riesgo ${ }^{127}$, cule constituye el único intento conocido por describir el posicionamiento del sector financiero español respecto de las recomendaciones del Comité de Basilea sobre la gestión del riesgo operacional. En él participaron 16 entidades financieras, bancos y cajas de ahorros, y se destacaba la existencia de dos grupos diferenciados. Un primer grupo que ya trabajaba activamente para alcanzar un enfoque avanzado y unos marcos de gestión en la línea de las entidades líderes internacionales. Y un segundo grupo de entidades, que aún estaban en İa fase inicial de análisis y definición para poder hacer frente a la gestión de este riesgo operacional. Este trabajo, además de poner de manifiesto el interés y la oportunidad del tema objeto de nuestra investigación en el contexto acual, nos permitió incorporar ciertos aspectos, que recogimos en el cuestionario, con el fin de contrastax algunos de los resuitados obtenidos por ellos.

\section{ASPECTOS METODOLOGICOS Y DISEÑO DEL ESTUDIO}

\subsection{Ondetrvos a Hurotesis}

Conscientes de que estábamos dando los primeros pasos ten conjunto, los trabajos sobre este tema fueron iniciados por las Cajas a comienzos de 2001), no quisimos entrar en aspectos que, siendo de gran interés en un futuro próximo, considerábamos prematuro ahondar en ellos, como después prueban los resultados obtenidos.

Por ello, los objetivos quie nos hemos propuesto conseguir, se centran en (1) analizar el grado de conocimiento de las entidades sobre el riesgo operacional, (2) evaluar la relevancia y el creciente interés por éste riesgo, y innalmente (3) valorar el grado de preparación y desarrollo de las entidades encuestadas de cara al futuro que plantea el Comité de Basilea.

Para alcanzar nuestros objetivos, las hipótesis que nos proponemos contrastar se pueden agrupar del siguiente modo.

La primera hipótesis básica (H1), formulada para contrastar el grado de identificación y consenso sobre el concepto de riesgo operacional, queda concretada en las dos hipótesis siguientes:

H1a. Todavia son muchas las entidades que no tienen asumida una definición formal de este riesgo.

H1b. Muchas entidades aún siguen identificando el riesgo operacional al riesgo operativo. 
La segunda hipótesis básica (H2), formulada para contrastar el grado de relevancia e interés alcanzado por el riesgo operacional, queda concretada en las dos hipótesis siguientes:

H2a. Las nuevas exigencias de los reguladores han sido la principal causa del aumento de interés por el riesgo operacional.

H2b. Existen otras causas del aumento de interés del riesgo operacional además de las puramente regulatorias.

Finalmente, Ia tercera hipótesis básica (H3), formulada para contrastar el nivel de desarrollo y preparación de las entidades para asumir el futuro que les espera en relación con este riesgo, queda concretada en la hipótesis siguiente:

H3. Aunque se requiere un esfuerzo importante para poder dar cumplimiento a las exigencias del Comité de Basilea, las entidades están trabajando para estar a la altura de las circunstancias en el 2007.

\subsection{Población y Muestra: El Sujeto}

Nuestra investigación empirica se centró en las Cajas de Ahorros. Una colaboración con la mesa de tesorería de la Confederación Española de Cajas de Ahorros (CECA) nos permitió tener acceso a las instituciones y a las personas que, seguras de nuestras confidencialidad, conocedoras del proyecto desde sus comienzos y conscientes del interés y complejidad del mismo, nos facilitarían la realización del trabajo con ciertas garantías de obtener unos resultados satisfactorios.

Por tanto, la población objeto del trabajo la configuran todas las Cajas de Ahorros de España, 46 instutuciones financieras, que representan casi la mitad de la cuota de mercado de los recursos ajenos, con el 46,45\% (datos a julio de 2002, CECA 2002), y que junto a las Cooperativas de Crédito han ido ganando posiciones en detrimento de la Banca. Esta población fue estratificada en función del tamaño, cuantificado por el total de sus recursos ajenos a 31 de julio de 2002, quedando finalmente conformada en los cuatro grupos que muestra la Tabla 2.

TABLA 2

DISTRIBUCTÓN DE LA POBIAGTON POR RECURSOS AJENOS

\begin{tabular}{|c|c|c|c|}
\hline & Cajas & $\begin{array}{c}\text { Recursos } \\
\text { que representan }\end{array}$ & $\%$ Recursos \\
\hline $\begin{array}{c}\text { GRupo A } \\
\text { (Más de } 10.000 \text { miliones } \\
\text { de euros de recursos } \\
\text { ajenos) }\end{array}$ & $\begin{array}{l}\text { C.E. I Pensions de BARCELONA } \\
\text { BILBAO BIZKALA KUTXA } \\
\text { C.E. de CATALUNAA } \\
\text { CAJA ESPANNA de Inversiones C.A.M.P. } \\
\text { C.A. de GALICIA } \\
\text { C.A. Y M.P. de MADRID } \\
\text { C.A. del MEDITERRANEO } \\
\text { UNICAJA } \\
\text { C.A. de VALENCIA, CASTELLON y ALICANTE } \\
\text { C.A.M.P. de ZARAGOZA, ARAGON y RIOJA }\end{array}$ & 234.492 & $63,29 \%$ \\
\hline
\end{tabular}


Tabla 2

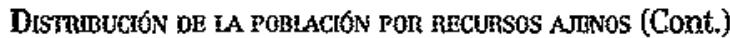

\begin{tabular}{|c|c|c|c|}
\hline & Cajas & $\begin{array}{c}\text { Pocursos } \\
\text { que representan }\end{array}$ & $\%$ Recursos \\
\hline $\begin{array}{c}\text { GRUPO B } \\
\text { (De 10.000 } \\
\text { a } 4.000 \text { millones de euros } \\
\text { de recursos ajenos) }\end{array}$ & $\begin{array}{l}\text { C.A. de ASTURIAS } \\
\text { C.A. de CASTIILLA LA MANCHA } \\
\text { C.A. y M.P. de CORDOBA } \\
\text { C.A.M.P. de GUIPUZCOA y S. SEBASTIAN } \\
\text { C.G. de A. de GRANADA } \\
\text { M.P. y C.A. de HUELVA-SEVILLA } \\
\text { G.A. de MURCIA } \\
\text { C.A. Y M.P. de NAVARRA } \\
\text { C.E. dOI PENEDES } \\
\text { C.A. y M.P. de SALAMANCA y SORIA } \\
\text { C. SAN FERNANOO DE SEVLLA Y JEREZ } \\
\text { C.A. te VIGO, OURENSE E PONTEVEDRA }\end{array}$ & 78.585 & $21,21 \%$ \\
\hline $\begin{array}{c}\text { GRupo C } \\
\text { (De } 4.000 \\
\text { a } 2.000 \text { millones de euros } \\
\text { de recursos ajenos) }\end{array}$ & $\begin{array}{l}\text { C.A. de la INMACULADA DE ARAGON } \\
\text { C.A. y M.P. de las BALEARES } \\
\text { C.A. WUnicIPal de BURGOS } \\
\text { C. GENERAL A. De CANARIAS } \\
\text { C.INSULAR A. de CANARIAS } \\
\text { G.A. y M.P. de EXTREMADURA } \\
\text { C.E. LAIETANA } \\
\text { G.E. de TARRAGONA } \\
\text { C.E. de TERRASSA } \\
\text { C.E. de SABADELL } \\
\text { C.A. de SANTANDER y GANTABRIA } \\
\text { C.A. de VITORIA y ALAVA }\end{array}$ & 41.747 & $11,27 \%$ \\
\hline $\begin{array}{c}\text { Grupo D } \\
\text { (Menos de } 2.000 \text { millones } \\
\text { de euros } \\
\text { de recursos ajenos) }\end{array}$ & $\begin{array}{l}\text { C.A. y M.P. de AVILA } \\
\text { M.P. y C.A. de BADAJOZ } \\
\text { C.A. y M.P. del C.C.O. de BURGOS } \\
\text { C.E. de GIRONA } \\
\text { C.A. Provincial de GUADALAJARA } \\
\text { C. Provineial de A. de JAEN } \\
\text { G.E. Gomarcal de MANULEU } \\
\text { G.E. de MANRESA } \\
\text { C.A. y M.P. de ONTINYENT } \\
\text { C.A. de POLLENSA } \\
\text { C.A. de Ia RIOJA } \\
\text { G.A. y M.P. de SEGOVIA }\end{array}$ & 15.676 & $4,23 \%$ \\
\hline & & 370,500 & $100,00 \%$ \\
\hline
\end{tabular}

FuENT: Boletin Estadistico do la CECA (2002) y elaboraçón propia (ordenación aliabética en cada grupo).

\subsection{METODOLOGiA UtIlizaDA}

Otley [1992], citando a Mitroff y Kilman, ya afirmaha que las distintas metodologías de investigación debían ser evaluadas en función de su capacidad para producir los resultados buscados. Nosotros, entre las distintas alternativas posibles de investigación empírica, he- 


\subsubsection{Estructura de los cuestionarios}

El cuestionario se configuró con un total de quince preguntas (ver anexo) diseñadas para conocer la opinión y estado de desarrollo respecto al riesgo operacional en las entidades que hemos elegido como sujetos de la investigación.

Los cuestionarios fueron enviados por correo en el mes de septiembre de 2002 a los directores o jefes de auditoría interna de las respectivas entidades, contactando con ellos posteriomente via e-mail y telefónicamente, dándose por finalizada la recogida de datos en diciembre de ese año. El motivo por el que decidimos elegir el departamento de auditoría interna como destino de nuestras encuestas es que, como ya señalábamos anteriormente y los resultados nos lo corroboran, el riesgo operacional afecta directamente a este departamento, siendo en la actualidad parte de sus funciones su establecimiento, evaluación, seguimiento y supervisión.

Las encuestas se plantearon totalmente anónimas y tan sólo se les pedía que marcaran a qué grupo pertenecía sui entidad en función del tamaño, siguiendo la clasificación que nosotros les proporcionábamos en una hoja adicional y que ya recogimos en la tabla anterior.

\subsubsection{Tratamiento de los datos}

Para el análisis estadístico ${ }^{(3)}$ hemos empleado fundamentalmente la estadística descriptiva para el resumen de la información univariable, tanto medidas de posición - distribuciones de frecuencias y los valores medios de las valoraciones-como medidas de dispersión - varianza y el coeficiente de Pearson-.

En algunas cuestiones nos interesó ir más allá de la información univariable, por lo que empleamos la inferencia estadística, estimando relaciones entre dos variables mediante matrices de correlaciones y tablas de contingencia, lo que nos permitió determinar tanto la condición de independencia como cuantificar el grado de asociación entre dichas variables a través del coeficiente de Pearson. Para asegurar si los valores y diferencias observadas son realmente significativas y no fortuitas, hemos aplicado pruebas de contraste de hipótesis, realizando la técnica Chi cuadrado, con la corrección de Yates, en aquellos casos en los que el número de respuestas no era lo sufieientemente grande. Así mismo, y para contrastar la posible existencia de relaciones vinculadas al tamaño de las Cajas, realizamos contrastes de Kruskal Waliis, que en caso de resultar no significativos nos permite extender y aplicar los resultados obtenidos a toda la población.

Junto a estas técnicas, y con el objeto de identificar la redundancia de la información recogida en las correspondientes escalas de Lickert, empleadas en la valoración de algunos de los aspectos planteados en el cuestionario, hemos aplicado el método de componentes principales, que constituye un caso particular de las técnicas multivariables globalmente conocidas como métodos factoriales. Dicha redundancia se verá reflejada en la correlación entre las distintas variables observadas y tendrá como resultado el reducir considerablemente el número de variabies sin que por ello se produzca una pérdida de información.

(3) Los fundarnentos teóricos en los que se basan nuestros resultados pueden verse, entre otros, en Bello et al. [1993] y Kinnear y Taylor [2000]. 


\subsubsection{Nivel de respuestas}

Las contestaciones recibidas, que se resumen en la Tabla 3, proporcionan un nivel de respuesta satisfactorio teniendo en cuenta el que por término medio se obtiene en este tipo de estudios y a lo novedoso del tema objeto de la encuesta. Además queremos agradecer la colaboración y disposición de todos aquellos que la respondieron, permitiendo con sus contestaciones el buen desarrollo de esta investigación.

\section{TABiA 3}

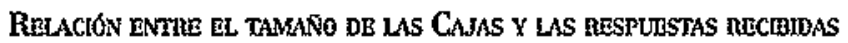
AL ESTUDIO DEL RIESCO OPRRACIONAL

\begin{tabular}{|l|c|c|c|c|c|}
\cline { 2 - 5 } \multicolumn{1}{c|}{} & Gaupo A & Grupo B & GaupD C & Gaupo D & \multicolumn{1}{c|}{} \\
\hline Enviadas & 10 & 12 & 12 & 12 & 46 \\
\hline Recibidas & 7 & 10 & 7 & 12 & 36 \\
\hline & $70 \%$ & $83 \%$ & $58 \%$ & $100 \%$ & $78 \%$ \\
\hline
\end{tabular}

En la Tabla 5 recogemos la ficha técnica que resume los datos del eşudio:

Tabla 4

FICha TÉCMLA DEL estudio

\begin{tabular}{|l|l|}
\hline Ambito & Espafia \\
\hline Poblacion & $\begin{array}{l}\text { Todas las Cajas de Ahorros y Montes de Piedad con sede y actividad en terri- } \\
\text { torio español (46 entidades) }\end{array}$ \\
\hline Sujeto & Directores de Auditoria Interna \\
\hline Muesira & $\begin{array}{l}\text { Tamaño: } 36 \text { Cajas te Ahorros } \\
\mathrm{E}= \pm 7,8 \% \quad \mathrm{NC}=95 \% \quad \mathrm{Z}=1,96 \quad \mathrm{p}=\mathrm{q}=0,5\end{array}$ \\
\hline Metodología & Cuestionario postal \\
\hline Trabajo de Campo & Septiembre - Diciembre de 2002 \\
\hline
\end{tabular}

\section{RESULTADOS DEL ESTUDIO EMPÍRICO}

\subsection{Conciztuación e Identificación del. Rifsgo Operacional.}

Según el estudio de PwC [2002: 10] la mayor parte de las entidades (63\%) han tomado como propia la definición de Basilea, tal y como se viene observando a nivel internacional, lo que ha servido de punto de partida para la homogenización de criterios y de un marco de referencia. Por eso, la primera pregunta que planteamos en el cuestionario fue: zcómo se define el riesgo operacional en su entidad?

Un $33,3 \%$ o no lo tenía formalmente definido o ni tan siquiera respondian la pregunta, lo que supone un resultado similar al obtenido por PwC (37\%). Sin embargo, a diferencia de lo detectado por ese trabajo, donde ninguna entidad adoptaba una definición diferente a

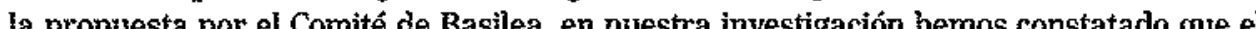


miten a los avances de la CECA y ai proyecto de riesgo global que ésta dirige, supone finalmente que sólo una cuarta parte de las entidades lo definen como lo hace el Comité de Basilea.

Como consecuencia de las exigencias de éste Comité, son muchas las entidades que o bien han modificado su definición $(24,2 \%)$, 0 al menos lo tienen previsto $(39,4 \%)$, pero nos interesaba conocer qué ocurría con el resto de las entidades. De ellas, que representan el $36,4 \%$, salvo una, todas las demás están de acuerdo con la definición del Comité de Basilea y se pueden clasificar en tres grupos. En primer ligar, dos entidades que no han modificado su definición porque han asumido directamente la del Comité, tres Cajas que aún no han tomado ninguna medida y están a la espera de los resultados del proyecto de la CECA, y el resto, siete Cajas, que teniendo su definición o sin tenerla, no se han planteado ninguna modificación. Suponemos que con el tiempo, acabaran por actualizar sus definiciones, pero esta actitud viene a reforzar la idea de que aún no hay la suficiente conciencia sobre este riesgo.

Esta situación confirma nuestra hipótesis $H 1 a$, ya que todavía hay muchas entidades que no tienen asumida ni recogida una definición formal de este riesgo, lo que nos induce a pensar que las Cajas de Ahorros, en su conjunto, están más próximas al segundo grupo de entidades que identificaba PwC [2002: 7], es decir, se encuentran inmersas en las fases iniciales de análisis y definición estratégica del riesgo.

Del análisis de esas definiciones propias que nos facilitan podemos concluir que, en primer lugar, salvo en una entidad que sigue adoptando una definición negativa del tipo: «el ries" go operacional es todo lo que no sea ríesgo de mercado ni de crédito», las demás dan definiciones positivas que describen lo que entienden por tal riesgo; y en segundo lugar, que en mayor o menor medida todas van en la línea de la definición del Comité de Basilea. De hecho así lo confiman el $74 \%$ de las entidades que manifiestan estar de acuerdo con dicha definición.

Sólo tres entidades declararon expresamente su desacuerdo con la definición propuesta, aunque una de ellas más que discrepar cuestionaba el hecho de que tal vez se esté definiendo un «saco» donde se dé cabida a todo riesgo, llegando incluso a confundirse con otros, tal y como exponía otra de las entidades que tampoco estaba conforme. Por otro lado la disconformidad de la tercera entidad se debia a la intención de considerar el riesgo legal como una categoría independiente.

La definición del Comité de Basilea, revisada y publicada nuevamente en febrero de 2003 [BASEL, 2003a], establece que: «Riesgo operacional es el riesgo de pérdidas como resultado de procesos intemos inadecuados o defectuosos, personal, sistemas o como resultado de acontecimientos externos. Se incluye el riesgo legal pero se excluye el riesgo estratégico, de reputación y sistémicon. Como se puede apreciar en la Tabla 5 , que recoge las definiciones aportadas por las entidades, distintas de la propuesta por el Comité de Basilea, mientras que todas incluyen dentro de su definición del riesgo operacional la posibilidad de incurrir en pérdidas como consecuencia de la operativa y de los procedimientos o procesos internos, los sistemas y el factor humano no están siempre presentes y en ningún caso se incluyen los eventos 0 acontecimientos externos como componentes de este riesgo. 


\section{Tamia 5}

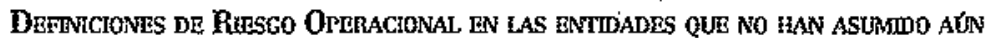
LA DETIICSON DEL COMTÉ DE BASHRA

\begin{tabular}{|c|c|c|c|c|c|}
\hline & \multicolumn{4}{|c|}{ PERDIDAS DEOIDA A } \\
\hline & & Sistemas & Procesos & $\begin{array}{l}\text { Errores } \\
\text { Humanos }\end{array}$ & $\begin{array}{l}\text { Factores } \\
\text { externos }\end{array}$ \\
\hline Def.1 & $\begin{array}{l}\text { Se corresponde con situaciones de pérditas } \\
\text { posibles derivadas de la ocurrencia de sucesos } \\
\text { inesperados o fallos en la operativa, sistemas y } \\
\text { procedimientos internos o externos. }\end{array}$ & $\sqrt{ }$ & & & \\
\hline Def.2 & $\begin{array}{l}\text { Posibilidad de incurrir en pérutitas por defieiencias } \\
\text { o ineficiencias de sistemas, personas o procesos. }\end{array}$ & $\checkmark$ & $\checkmark$ & $\checkmark$ & \\
\hline Def.3 & $\begin{array}{l}\text { Quebrantos y costes de oportunidad en las que } \\
\text { puede lncurnir la Entidad, derivados de la operativa } \\
\text { ordinaria y de la wilnerabilidad de los } \\
\text { procedimientos y normativa interna que estén } \\
\text { establecidos en la mlsma. }\end{array}$ & & & & \\
\hline Def. 4 & $\begin{array}{l}\text { Riesgo que se incurre al operar: fuera de los } \\
\text { procedimientos establectidos, por fallos en las } \\
\text { transacciones y aplicaciones, con productos no } \\
\text { autorizados, desconocimiento por parte del } \\
\text { personal o errores en la información contable. }\end{array}$ & $\mathscr{V}$ & $\checkmark$ & $\checkmark$ & \\
\hline Def.5 & $\begin{array}{l}\text { Riesgo derivado de sucesos inesperados } \\
\text { relacionados con la infraestructura humana } \\
\text { y tecnológica o con los procedimientos establecidos. }\end{array}$ & $\checkmark$ & $\checkmark$ & $\sqrt{ }$ & \\
\hline Def.6 & $\begin{array}{l}\text { Riesgo de pérdidas por fallos en los procesos } \\
\text { utilizados. }\end{array}$ & & $\checkmark$ & & \\
\hline Def.7 & $\begin{array}{l}\text { Probatilidad de sufrir pérdidas por la reatización o } \\
\text { por la ocurrencia de fallos en los procesos } \\
\text { o sistemas. }\end{array}$ & $\checkmark$ & $\checkmark$ & & \\
\hline Def.8 & $\begin{array}{l}\text { El riesgo al que se expone la enttdad como } \\
\text { consecuencia de acontecimientos inesperados o } \\
\text { errores derivados de la operativa ordinaria y da la } \\
\text { transgresión de ta normativa interna } \\
\text { y procedimientos. }\end{array}$ & & $\sqrt{ }$ & & \\
\hline Def. 9 & $\begin{array}{l}\text { Posibilidad de pérdidas provocadas por fallos en } \\
\text { los procesos operativos, en ios sistemas o en los } \\
\text { recursos humanos que los soportan. }\end{array}$ & $\checkmark$ & $\checkmark$ & $\sqrt{ }$ & \\
\hline
\end{tabular}

En previsión de aquellas entidades que no tuvieran una definición expresa, y para facilitar su respuesta, les preguntamos cirales de entre una serie de subcategorías que les proponiamos incluían en su interpretación del riesgo operacional. De los resultados obtenidos, recogidos en el Gráfico 1, se aprecia que el riesgo legal es una categoría independiente de riesgo en el $75 \%$ de las entidades, mientras que el Comité de Basilea sí lo incluye, y por el contrario el riesgo de reputación, excluido expresamente de la definición del Comité, sí ha sido considerado por cuatro entidades como parte del riesgo operacional (11\%). 


\section{Grafico 1}

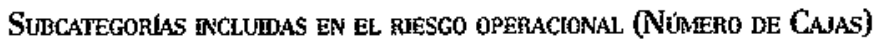

Riesgo estratégico o de negocio

Riesgo de reputación o de que se dañe la imagen

Riesgo sistémico

Fiesgo legal

Riesgo de tomar decisiones inadecuadas conno consecuencia de una información incorre

Riesgo de fraudes internos o externos

Riesgos asociados a la tecnoiogla empleada o tas aplicaciones informáticas implantadas

Riesgo de fallo en los controles internos

Riesgo de péroida por la realización de actividades no autorizadas

Riesgo de errores en los registros contrables

Riesgo de que el persenal no trabaje correctamente o cometa fallos

Riesgo de que se ejecuten incorrectamente las transacciones

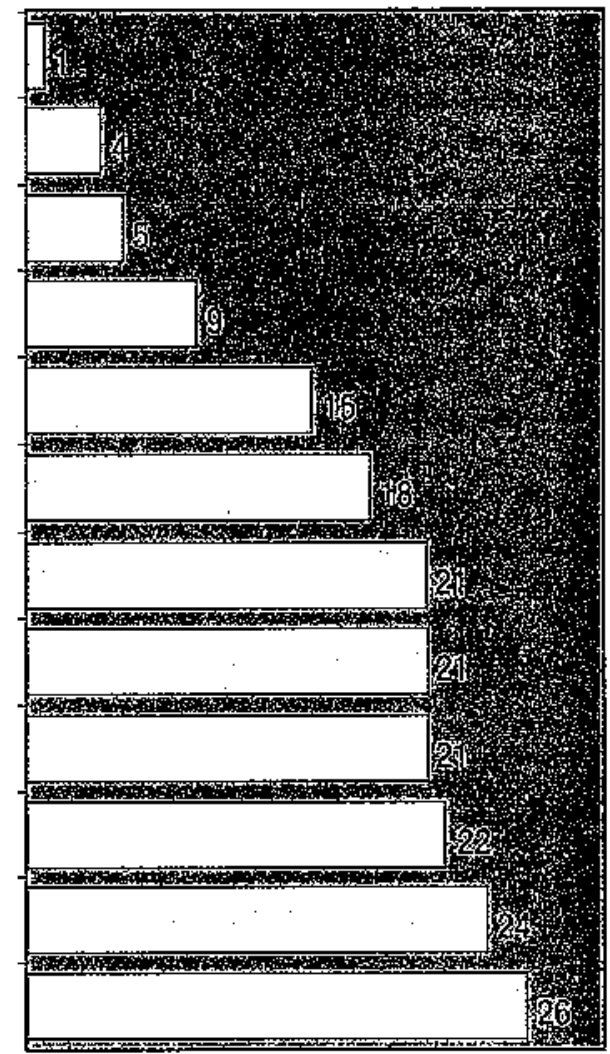

En definitiva, y contrastándose positivamente la hịótesis $H 1 b$, pødemos afirmar que muchas entidades aún siguen identificando el riesgo operacional al riesgo operativo y por $\tan ^{n}$ to, se sigue confundiendo y equiparando con el riesgo que es debido únicamente a las operaciones que realiza la entidad.

\subsection{Relevancia y Aumento del Intertes por el Ruesco Operacional}

En los últimos años el interés por el riesgo operacional ha aumentado muy considerablemente [BBA, RMA, ISDA, 1999]. Nos cuestionamos si para las Cajas de Ahorros, como lo fue para las entidades del estudio de PwC [2002: 10] en su conjunto, el principal motivo por el que ha crecido el interés por el tema ha sido la aparición de este nuevo entorno regulatorio marcado por los avances del Comité de Basilea o, si por el contrario, hay otras cazsas que han incidido en mayor medida sobre este hecho.

Para ello les solicitamos que valorasen la importancia de las nueve causas que a muestro juicio pueden haber influido sobre el creciente interés y que se recogen en la pregunta 7 del cuestionario. 
Analizando los valores medios de las respuestas recibidas, representados en el Gráfico 2, comprobamos lo que sucede con las hipótesis $H 2 a$ y $H 2 b$. Por un lado, efectivamente el Nuevo Acuerdo de Capital de Basilea $\left(C_{1}\right)$ y la creciente atención de los reguladores $\left(C_{8}\right)$ son los dos factores principalos de este creciente interés, confirmándose positivamente H2a ya que se demuestra que las nuevas exigencias de los reguladores than sido la principal causa del aumento de interés por el riesgo operacional. Pero por otzo lado, el aumento percibido en este riesgo $\left(C_{2}\right)$, la nueva tendencia de gestión integral del riesgo $\left(C_{7}\right)$ y el interés por controlar y reducir las pérdidas operacionales $\left(C_{5}\right)$ son también otros de los factores señalados como causas relevantes del aumento de dicho interés, confirmándose de nuevo positivamente nuestra hipótesis $H 2 b$.

Asimismo, comprobamos que realmente las entidades aún no son conscientes de las pérdidas que pueden tener, y de hecho se originan por este riesgo, o al menos no identifican sus efectos, ya que esa causa $\left(C_{3}\right)$ queda posicionada en último lugar:

\section{GRAFICO 2}

VALORAGION DE IAS CAUSAS QUE HAN INTLUIDO RN RL GRECERTE INTBRés

C1 Las exigencias de captital por riesgo operacional del Nuevo Acuerdo de Capital de Basilea.

Ca La creciente atención de los reguladores sobre este riesgo.

Ca El aumento percibido de este riesgo, consecuencia de la globalización, creciente complejidad instrumentos, nuevos mercados, e-business, etc.

G. La nueva tendencia de gestión integral del riesgo cono secuencia lótgiea después de abordar el de mercado y dee crérito.

Cs Interés por controlar y seducir pérdildas operacionales.

C9 La concienciación de la alia direcetón con este rtesgo.

C4 Las Importantes pérdidas suffidas por otras entioades atribuibles a este riesgo.

Co Búsqueda de ventajas competitivas.

$\mathrm{C}_{3}$ Las importantes pérdidas suffidas internamente en la entidad atribuibies a este riesgo.

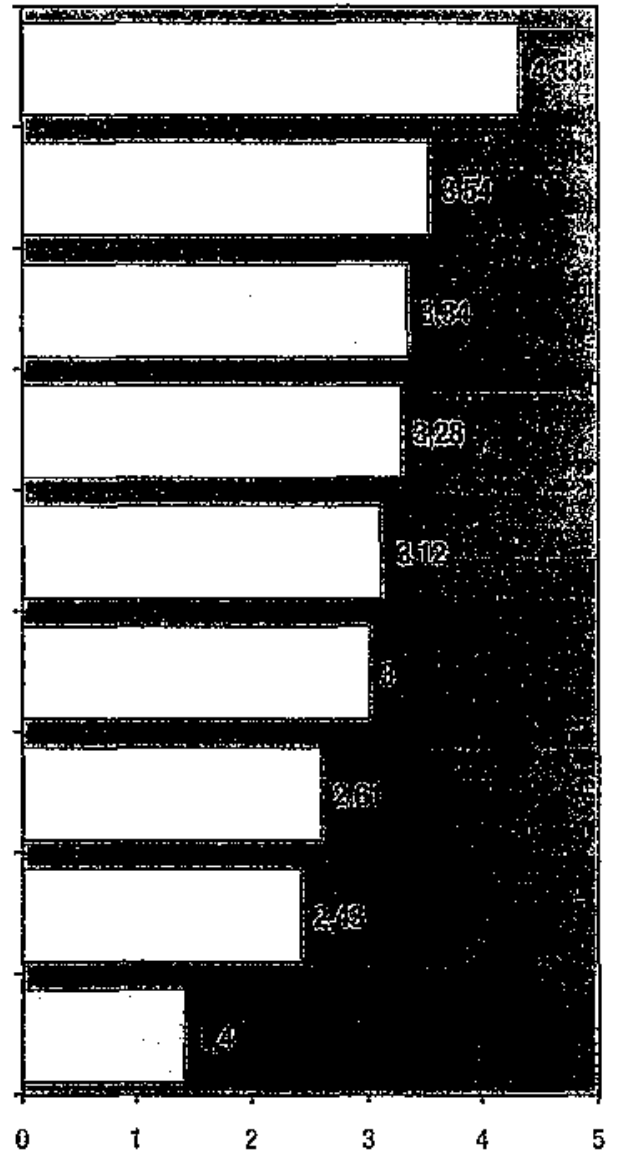


Con el fin de conocer la existencia de alguna pauta de comportamiento que resumiera mejor las actitudes de las entidades ante las causas que propician la importancia y el interés por el riesgo operacional, hemos realizado una reducción factorial mediante el análisis de los componentes principales, eliminando previamente la causa $C_{3}$ comentada, ya que su baja representatividad distorsionaba las conclusiones. El resultado, recogido en la Tabla 6, ha sido que el valor del determinante de la matriz de correlaciones se encuentra próximo a cero, lo que indica una elevada asociación entre las ocho causas identificadas. Este resultado es consistente con el test de esfericidad de Bartlett, cuyo valor llevado a la distribución Chi cuadrado permite rechazar la hipótesis de que la matriz de correlaciones sea equivalente a la matriz identidad. Además el índice KMO presenta un valor también próximo a cero, lo que refuerza los resultados de las dos priebas anteriores. En consecuencia, se puede afirmar la posibilidad de identificar estructuras latentes en este conjunto de causas, justificada por los niveles de asociación observados.

TABZA 6

ÍNEICES DE VALIDEZ DEL ANÁLISTS DE LOS COMPONENTES PRINCJPALES DB LAS CAUSAS DEL INGREMIENTO PERCIBIDO EN EL. INTERESS SOBRE EL RIESGO OPERACIONAL

\begin{tabular}{|c|c|}
\hline Indices & Valores \\
\hline Determinante de la matriz de corralaciones & 0.146 \\
\hline Medida de adecuación muestral de Kaiser-Meyer-Okin & 0.557 \\
\hline Prueba de esfericidad de Barlett & \\
\hline Chi cuadrado & 49.076 \\
\hline Grados de libertad & 28 \\
\hline Significación & 0.008 \\
\hline
\end{tabular}

Para la extraccíon de Ios componentes principales, Tabla 7 y Tabla 8, se optó por el criterio de los valores propios mayores que la unidad, obteniéndose cuatro factores que explican, en su conjunto, el $78,235 \%$ de la varianza de las observaciones.

TABLA 7

AUTOVALORES $\times$ \% VARIANZA EXPLICADA EN El ANALISIS dE LAS CAUSAS

\begin{tabular}{|c|c|c|c|}
\hline Componente & Autovalor & \% de la varianza & \% acumulado \\
\hline 1 & 2,32 & 29,10 & 29,10 \\
\hline 2 & 1,58 & 19,77 & 48,87 \\
\hline 3 & 1,34 & 16,78 & 65,65 \\
\hline 4 & 1,00 & 12,59 & 78,25 \\
\hline 5 & 0,58 & 7,32 & 85,57 \\
\hline 6 & 0,52 & 6,57 & 92,15 \\
\hline 7 & 0,37 & 4,65 & 96,81 \\
\hline 8 & 0,25 & 3,18 & 100,00 \\
\hline
\end{tabular}




\section{TABLA 8}

Cargas ractorlales do los componentes princtpales rotados en las causas del aumento dei mierés

\begin{tabular}{|c|c|c|c|c|}
\cline { 2 - 5 } \multicolumn{1}{c|}{} & Componente 1 & Componente 2 & Componente 3 & Componente 4 \\
\hline $\mathrm{C}_{1}$ & 0,778 & $-0,259$ & 0,013 & 0,234 \\
\hline $\mathrm{C}_{2}$ & 0,356 & 0,078 & 0,808 & $-0,002$ \\
\hline $\mathrm{C}_{4}$ & $-0,388$ & $-0,182$ & 0,803 & 0,043 \\
\hline $\mathrm{C}_{5}$ & 0,216 & 0,791 & 0,015 & $-0,354$ \\
\hline $\mathrm{C}_{6}$ & $-0,163$ & 0,803 & $-0,130$ & 0,291 \\
\hline $\mathrm{C}_{8}$ & 0,818 & 0,104 & $-0,076$ & 0,028 \\
\hline $\mathrm{C}_{6}$ & 0,159 & 0,003 & 0,033 & 0,919 \\
\hline $\mathrm{C}_{9}$ & 0,692 & 0,479 & 0,280 & $-0,058$ \\
\hline
\end{tabular}

La interpretación de los componentes nos lleva a identificar a cuatro tipos de entidades que son explicados por dos o tres de las causas restantes salvo el cuarto que viene dado por un ínico componente.

Así tenomos en primer lugar a aquellas entidades cuyo interés por el riesgo operacional se debe principalmente a las nuevas exigencias de capital del Comité de Basilea, que obligan a concienciar a la alta dirección de la necesidad de gestionar el riesgo operacional del mismo modo que previamente se hiciera con los riesgos de crédito y de mercado.

En segundo lugar, están las entidades que ven en la gestión del riesgo operacional una fuente de ventajas competitivas frente al mercado, al permitir con ello controlar y reducir las pérdidas operacionales. Su motivación es puramente interna.

En tercer lugar, están las entidades que, arrastradas por un entorno donde se ha percibido un aumento de este riesgo truto de la globalización y complejidad de los mercados han visto como se han incrementado también las pérỏidas por este riesgo. Su motivación la encuentran fuera de su entidad. Es por tanto un factor exógeno la causa de su interés.

Finalmente, tenemos un cuarto grupo de entidades que han experimentado un interés creciente por este riesgo debido fundamentalmente a la creciente atención de los reguladores, tanto nacionales como internacionales.

En resumen, se confirman positivamente las hipótesis $H 2 a$ y $H 2 b$ y podemos concluir que aunque las recientes exigencias de los reguladores, y muy especialmente del Comité de Basilea, han motivado a las entidades a fijar su atención sobre el riesgo operacional, ésta no ha sido la única causa del fenómeno, ya que, entre otras, el crecimiento percibido en este riesgo así como las nuevas tendencias de gestión que obligan a su consideración con el objeto de reducir y controlar las pérdidas operacionales por él sufirias han contribuido igualmente al aumento de interés percibido sobre el riesgo operacional.

\subsection{Desarrollo y avances en el Riesgo Operactonal}

\subsubsection{Actitud ante los requerimientos de Basilea}

Aunque pueda parecer que llegas a definir el riesgo operacional es una tarea trivial o po- 
preocupa en gran medida a las entidades. En el estudio de PwC se destacaba que la opinión acerca de los nuevos requerimientos del Comité de Basilea era neutra $(43,75 \%)$, o positiva $(31,25 \%)$, ya que aunque indudablemente la implantación supone un importante es. fierzo, las mejoras en los procesos de gestión y asignación de capital son evidentes. En este sentido, nuestra investigación constata que aunque sólo un $6 \%$ de las Cajas es rotundamente contrario a las nuevas exigencias, la mayoría de estas instituciones, aun estando de acuerdo, creen que es demasiado pronto e incluso excesivo el porcentaje de capital regulatorio previsto por el Comité de Basilea para cubrir este riesgo, y eso a pesar de que éste ya fue reducido en el documento de septiembre de 2001 «Working Paper on the Treatment of Operational Risk» [BASEL 2001b] ${ }^{(4)}$.

\subsubsection{Medidas adoptadas para la gestión del riesgo operacional}

Como consecuencia de todo este proceso un $53 \%$ de entidades ya han tomado algún tipo de medida, incluso desde 1999, y un 35\% tenía previsto hacerlo a lo largo del año 2002 y en los años sucesivos. Entre las medidas adoptadas, la más importante es la participación como Caja piloto en el módulo de riesgo operacional del proyecto sectorial de gestión y control global del riesgo de la CECA. Dicho proyecto, en el que participan la mayoría de las Cajas de Ahorros, tiene como fin, entre otros, el desarrollo e implementación de herramientas comunes de medición y control del riesgo operacional adenós de la creación de una base de datos de pérdidas operacionales que pueda servir a todo el colectivo. Queremos destacar la importancia de este tipo de iniciativas ya que de no ser por ellas y otras similares, muchas entidades de pequeña y mediana dimensión nunca podrían alcanzar los niveles adecuados de desartollo por su elevado coste.

Como ya indicábarnos, el grado de avance del sector financiero español en la gestión del riesgo operacional, a la fecha de este estudio, es todavía bastante limitado, encontrándose án muy alejado de los niveles alcanzados en otros riesgos como el de crédito y de mercado. Además, debemos añadir el desigual desarrollo que están experimentando las entidades entre sí. Así, por ejemplo, todavía no hay ninguna Caja que cuente con un comité que centralice las discusiones y procesos de toma de decisiones a nivel corporativo para este riesgo, y tan sólo dos Cajas tienen un área independiente de gestión del riesgo operacional, aunque ocho lo estén desarrollando; resultados que, cono se recoge en los Gráficos 3 y 4, discrepan de los obtenidos entre las entidades líderes del estudio de PwC.

La raíz de estas discrepancias consideramos que está, al igual que como señalábamos a la hora de identificar la definición de riesgo operacional, en que las Cajas se encuentran todavía en las fases previas del proceso, delimitando y definiendo el problema. Por el contrario, en el trabajo de PwC había también un grupo de entidades que llevaba más tiempo trabajando activamente para aicanzar un enfoque avanzado y ya están desarrollando un marco de gestión en la línea de las entidades líderes a nivel internacional.

(4) Inicialmente el Comité de Basilea, basảndose en los datos aportados por una muestra de bancos y otras fuentes, estableció la cifra de capital regulatorio por Riesgo Operacional, para el modelo básico, en un 20\% de sus recursos. Sin ermbargo, después de las fuertes criticas y quejas recibidas por el sector, que lo consideraba excesivo, y para ello argumentaba que la muestra empleada por el Comité no era representativa por su reducido tamaño y que además que con dicho porcentaje se acabaría incrementando el nivel general de exigencias de capital, contrario a los objetivos del Comité, se redujo en el working paper de septiembre de 2001 hasta un $15 \%$, en el método básico, y un $12 \%$, para aigunas de ias líneas en ef método estándar. 
Gurico 3

EXISTENGI DE UN ÁREA INDDPENDENTE DE GESTION DEL, MESGO OPRRACIONAL

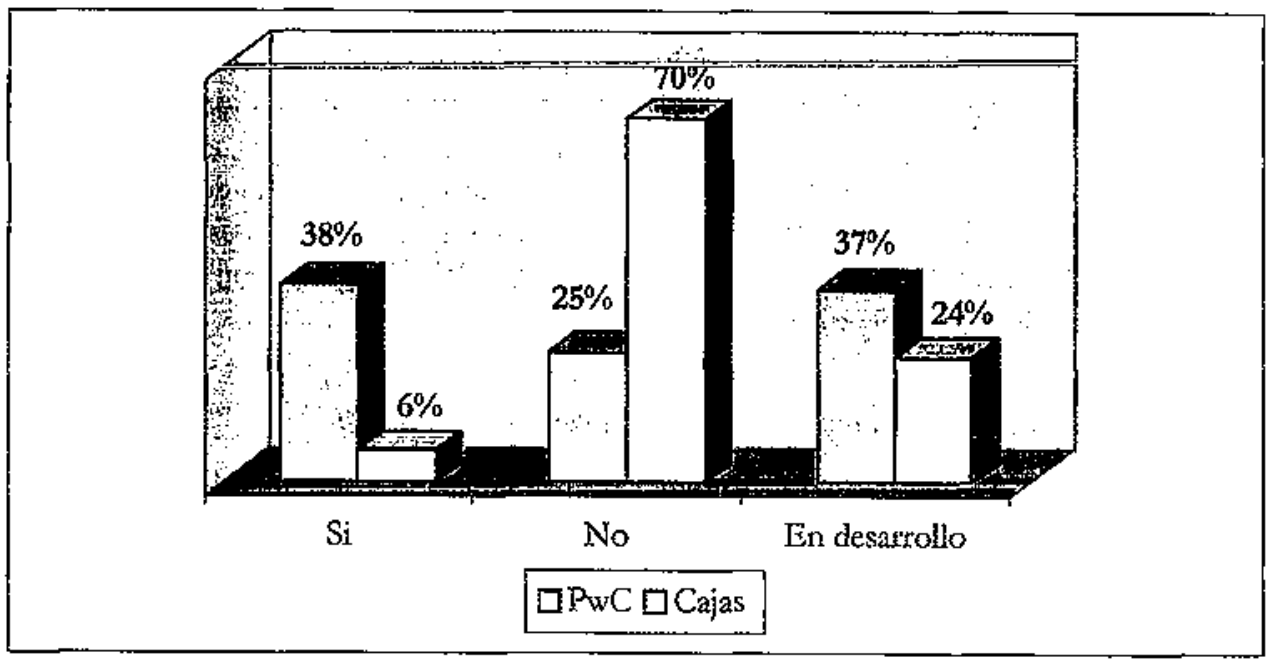

GRAFCO 4

EXISTENCIA DE UN COMTTÉ DE GESTION DEL RRSGO OPERACIONAL

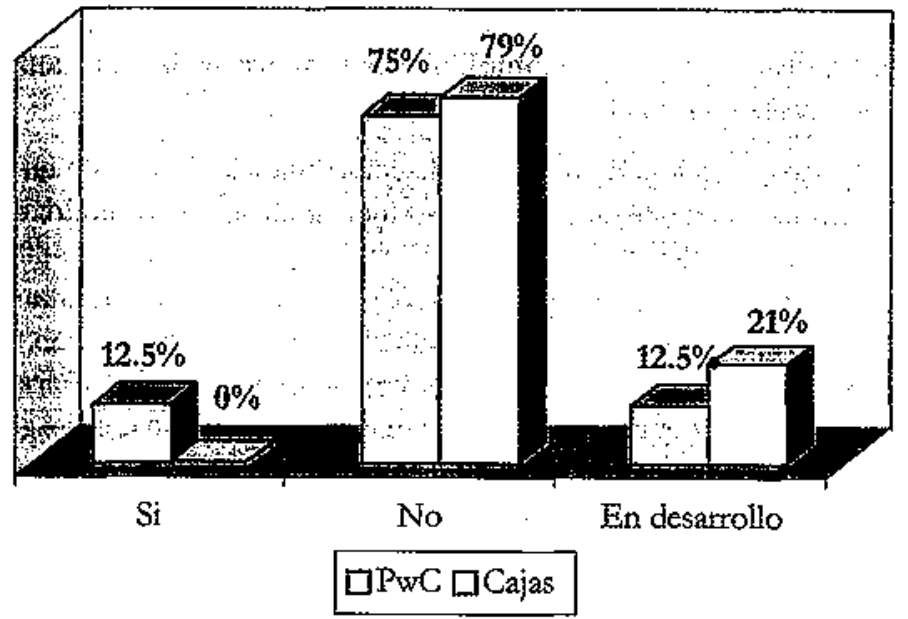

Uno de los elementos esenciales para poder gestionar y cuantificar el riesgo operacional es la disponibilidad de datos internos de pérdidas, motivo por el cual el Conité de Basilea insta a las entidades a empezar a capturar estos datos, que hasta ahora no se recogían, y llegar a compararlos con el sector. Aquí se aprecia nuevamente el precario estado en el que aún se encuentran algunos de los pilares para la gestión del riesgo operacional, pues tan sólo una Caja, que además resulta ser del grupo D, cuenta ya con una base de datos de pérdidas operacionales, mientras que la mayoría ni la tienen ni está en desarrollo, resultados que nuevamente discrepan en buena medida de los avances alcanzados por las entidades pioneras en el sector, como se refieja en el Gráfico 5 , donde el $18 \%$ tiene una base de da- 


\section{GRÁFCO 5}

EXISTENCTA DE UNA BASE DE DATOS DE PERDIDAS OPERACIONALES

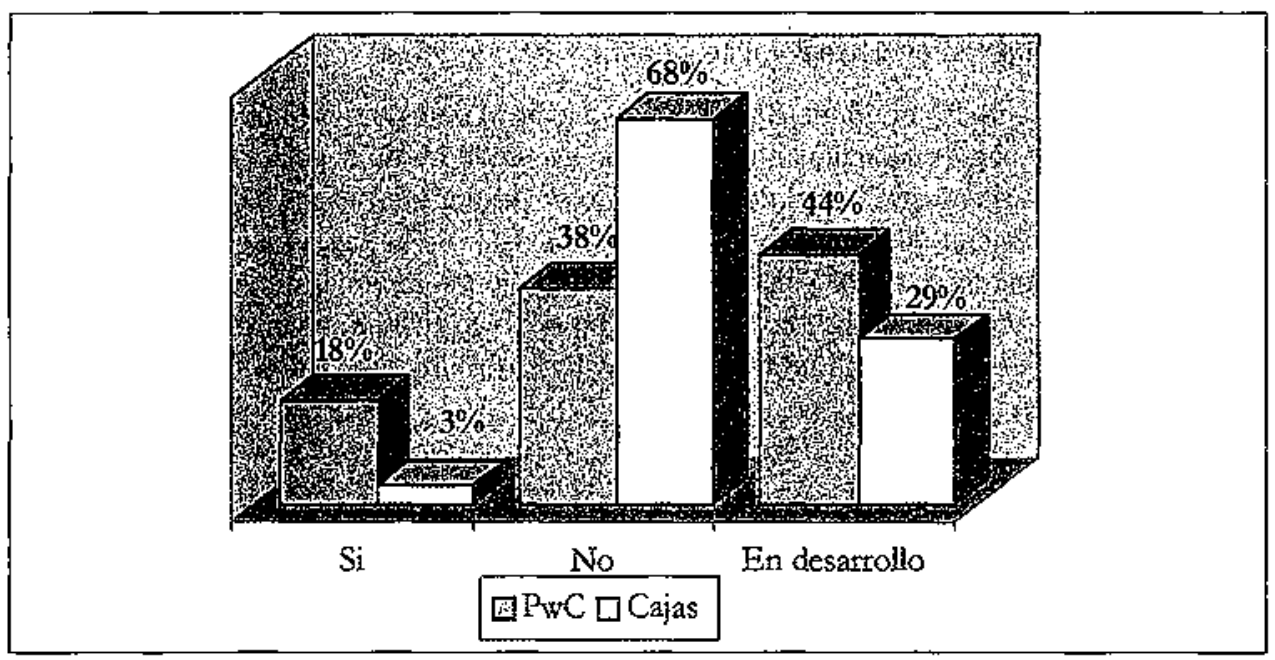

\subsubsection{El papel del departamento de auditoría interna}

Como adelantábamos anteriormente es este estado inicial en el que nos encontramos lo que hace que el departamento de auditoria interna aún juegue un papel muy importante llevando la gestión y el control de este riesgo. Este papel, concretado en las funciones de seguimiento y supervisión del riesgo operacional, es más acusado todavía en el caso de las Cajas, como se refleja en el Gráfico 6, como consecuencia de que el grado de desarrollo de otras áreas específicas para la gestión del riesgo operacional está menos avanzado que en algunos de los bancos incluidos en el estudio de PwC.

\section{GRAFICO 6}

\section{Papel de la AudTronía INterna}

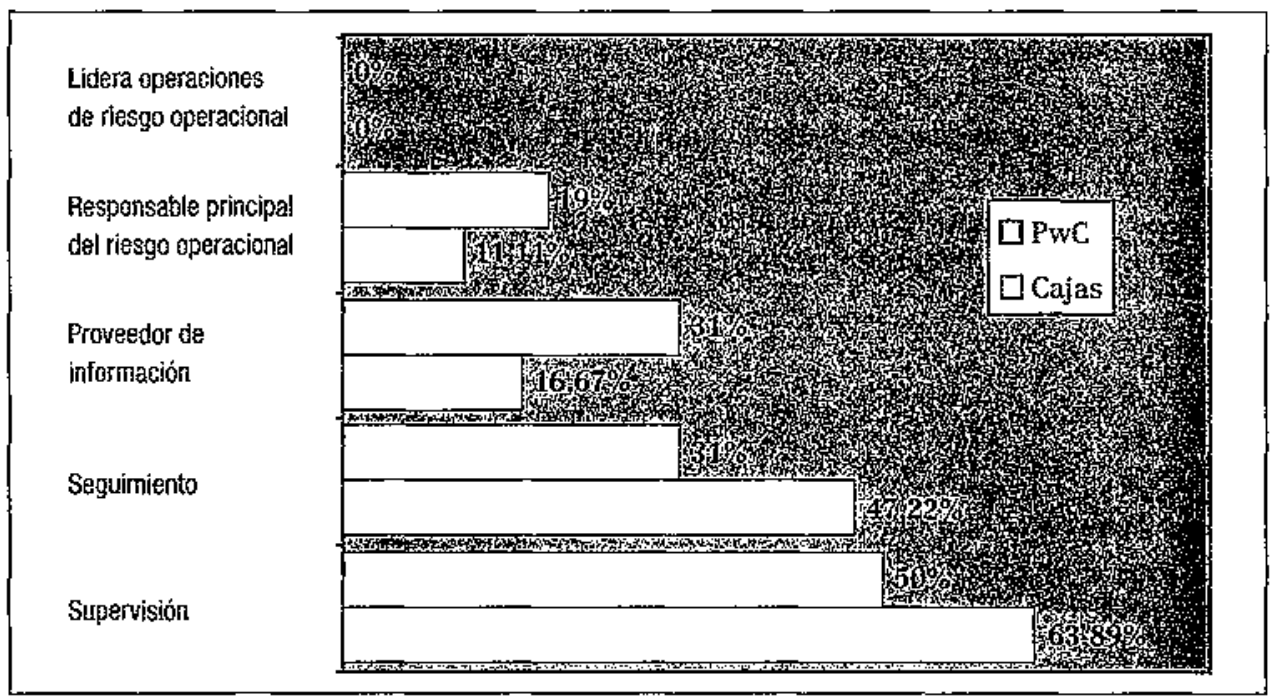


Suponemos que esta situación se mantendrá transitoriamente, ya que recordemos que el Nuevo Acuerdo de Basilea limita el papel de la auditoría interna a la supervisión de las políticas y los procedimientos establecidos, eximiéndola de un papel activo en la gestión de este riesgo para evitar potenciales conflictos de interés.

En la mayor parte de las entidades la gestión y el control de este riesgo no está asignado a nadie de forma específica o lo llevan cada una de las unidades de negocio. Sin embargo, ya hay entidades donde es misión del departamento de gestión de riesgos o del departamento de riesgo operacional en los casos en los que ya existe. En la Tabla 9 se recogen los encargados de la gestión, control y supervisión del riesgo operacional en las entidades encuestadas, donde nuevamente se comprueba que el único resultado claro y rotundo es el papel supervisor de la auditoría interna porque el resto de los resultados está tan disperso como las ideas.

\section{TABIA 9}

FUNCIONES RELACTONADAS CON EL RIESGO OPERACIONAL̃

\begin{tabular}{|c|c|c|c|c|c|c|c|}
\hline & $\begin{array}{c}\text { DPYO. OE } \\
\text { GESTION } \\
\text { DE RIESGOS }\end{array}$ & $\begin{array}{c}\text { DPTO. OE } \\
\text { AUOHORIA } \\
\text { INIERAA }\end{array}$ & $\begin{array}{c}\text { DPrO. DE } \\
\text { RiESGO } \\
\text { OPEAACOOHAL }\end{array}$ & $\begin{array}{c}\text { CADA UHA OE } \\
\text { LAS UWHDAOES } \\
\text { DE NEGOCSO }\end{array}$ & $\begin{array}{c}\text { DE FORMA } \\
\text { ESPECIFICA } \\
\text { NADIE }\end{array}$ & $\begin{array}{l}\text { AUUN NO } \\
\text { ESTA } \\
\text { PREUSTO }\end{array}$ & Otros \\
\hline Gestion & 7 & 5 & 1 & 10 & 7 & 3 & 2 \\
\hline Control & 7 & 8 & 4 & 3 & 8 & 4 & 3 \\
\hline Supervisión & 2 & 23 & 0 & 2 & 5 & 2 & 3 \\
\hline
\end{tabular}

\subsubsection{Grado de preparación para el futuro}

Finalmente, quisimos valorar hasta que punto se veían las entidades preparadas en ese momento para hacer frente a los cambios y las recomendaciones que propone el Comité de Basilea, para lo que les pedimos su auto evaluación de 1 a 10. De los resultados obtenidos, debemos destacar, en primer lugar, que la media de todo el colectivo de Cajas no lega al «aprobado», con una valoración conjunta de 4,39 y, en segundo lugar, el hecho de que se vean menos preparadas aquellas entidades más grandes, que en principio se suponen mejor preparadas. A pesar de todo ello, reforzadas por el proyecto de riesgo global liderado por la CECA, el $88 \%$ de las Cajas creen que estarán preparadas para poder aplicar alguno de los métodos de asignación de capital para cubrir el riesgo operacional propuestos por Basilea para el 2007, aunque la mayoría, el $52 \%$, no tengan todavía previsto cual de ellos va a ser el que adopten en su momente, a diferencia de las 16 entidades de PwC que ya habian optado por un método, en su mayoría el avanzado. 


\section{Grafrco 7}

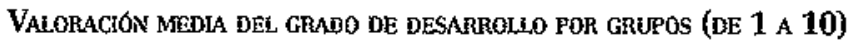

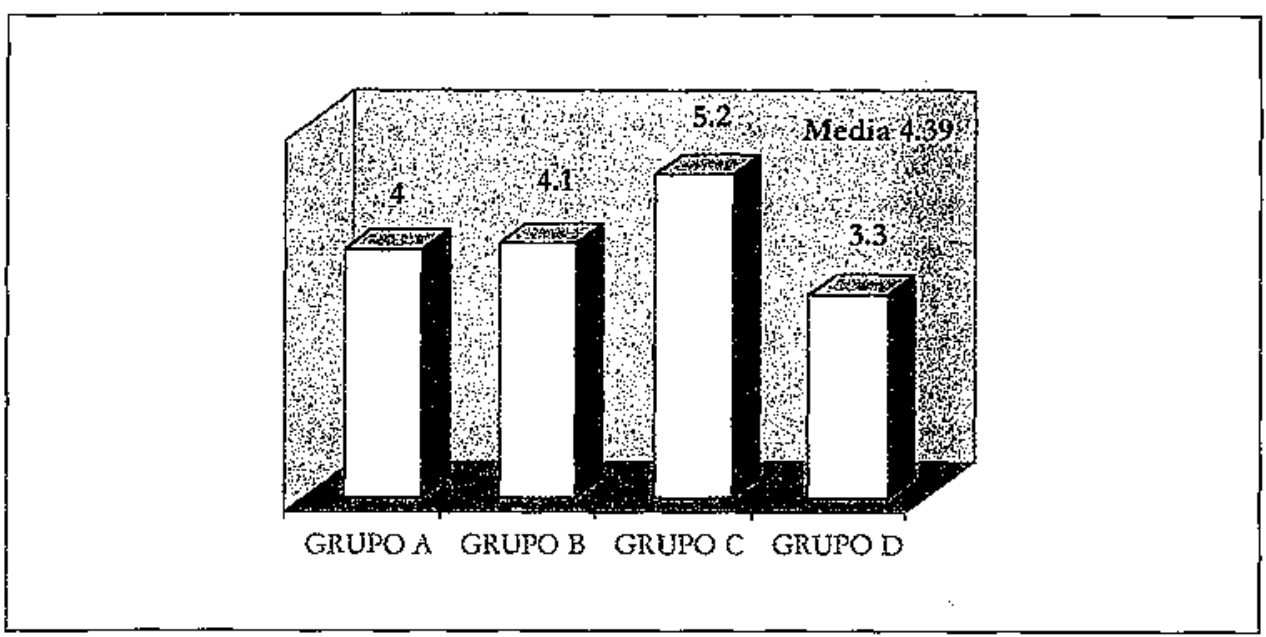

\section{GRAFCO 8}

MÉTODO DE ASIGNACTÓN DE CAPTTAL PREVISTO EN SU ENTMAD

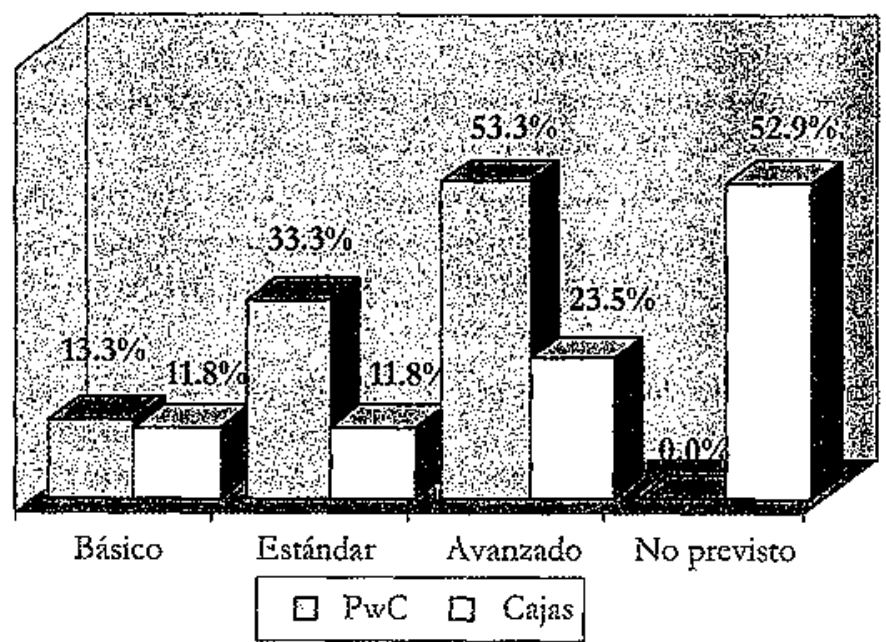

En definitiva, todos estos resultados confirman positivamente nuestra última hipótesis H3 ya que aunque se requiere un esfuerzo importante para dar cumplimiento a las exigencias del Conité de Basilea, las entidades están trabajando para estar a la altura de las circunstancias en el 2007, y el sector financiero español, en particular las Cajas de Ahoros, ayudadas e impulsadas por la CECA, estarán preparadas para afrontar la nueva situación. 


\section{CONCLUSIONES}

Si bien el riesgo operacional no es algo nuevo, en los últimos años estamos asistiendo al creciente interés de las entidades, en especial las financieras, y en particular las Cajas de Ahorros, por crear y desarrollar un marco que les permita su gestión global. Sin embargo, el grado de avance es todavía bastante limitado, encontrándose muy alejado de los niveles alcanzados en otros riesgos como el mercado o de crédíto. Las Cajas de Ahorros no están suficientemente preparadas o no disponen de toda la información necesaria sobre este riesgo, lo que nos induce a pensar que, en su conjunto, están inmersas en las fases iniciales de análisis y definición estratégica de este riesgo.

El Nzzevo Acuerdo de Capital de Basilea, que por primera vez exige que se dote capital por este riesgo, y el interés creciente de los reguladores son las causas principales que han empujado a las Cajas de Ahorros a preocuparse por el riesgo operacional, como previamente lo habian hecho con otros riesgos, colocándolas a la vanguardia de su gestión en comparación con otros sectores. Aunque hemos podido comprobar que existen otras causas ademấs de las puramente regulatorias, las entidades, aun percibiendo las pérdidas suiridas por otras entidades como consecuencia de este riesgo, no son conscientes, 0 al menos no identifican en su propia organización, los efectos y las pérdidas que pueden tener por una gestión de este riesgo inapropiada.

Con una definición de riesgo operacional recientemente consensuada dentro del propio sector bancario, muchas Cajas de Ahorros, aún no la tienen asumida de manera formai y ello puede propiciar que se siga confundiendo con el riesgo operativo, limitado a las pérdidas derivadas de las operaciones y los procesos. Por tanto, en el ámbito de estas entidades parece que todavía son bastantes los aspectos que deben ser desarroliados para hacer frente con éxito a las exigencias del Conité de Basilea en el 2007.

Por otra parte, dada la situación inicial que atraviesan y hasta que sea alcanzado el nivel de desarrollo deseado, el papel de los departamentos de auditoria interna está siendo determinante, siendo los encargados de impulsar y llevar las iniciativas necesarias para la gestión del riesgo operacional.

Finaimente y a pesar de que los avances se están produuciendo con gran rapidez, las Cajas de Ahorros, ayudadas por las iniciativas de los reguladores, legisladores, instituciones y muy especialmente por la CECA, aún tienen un largo camino por recorrer, en el que confiamos baber contribuido con el desarrollo de este trabajo.

\section{BIBLIOGRAFIA}

AFRT, L. [2001]: «A framework for managing operational risk», The Internal Auditor Vol. $58, \mathrm{n}^{\circ} 4$, pp. 53-59.

ANTHONy, R. [1990]: El control de gestión, (Bilbao: Deusto).

ANDrrsEN, A. [1998]: Operational risk and financial institutions, (Arthur Andersen, London: Risk Books).

BASEa. COMMTTEE ON BANkING SuPERusion [1998]: Operational risk management, BASEL, Basilea. (online) http://www bis.org

- [1999]: A new capital adequacy framework, BASEL, Basilea. (online) http:/fwww.bis.org. 
BaSEL COMMTTEE ON BANKang SUPBRnSION [2001a]: Operational risk (A consultative paper), BASEL, Basilea, (online) http://www.bis.org.

- [2001b]: «Regulatory treatment of operational risk», Working Paper, BASEL, Basilea, (online) http://wwwbis.org.

- [2001c]: Sound practices for the management and supervision of operational risk, BASEL, Basilea, (online) http://wwwbis.org.

— [2001d]: QIS 2: Operational risk data, BASEL, Basilea, (online) http://www.bis,org,

- [2002]: Sound practices for the management and supervision of operational risk, BASEL, Basilea, (online) http://wwwbis.org.

- [2003a]: Sound practices for the management and supervision of operational risk, BASEL, Basilea, (online) http://wwwbis.org.

- [2003b]: New Basel Capital Accord (Third Consultative paper, CP3), BASEL, Basilea, (online) http://www.bis.org.

- [2004]: Basel II: International Convergence of Capital Measurement and Capital Standards: $\alpha$ Revised Framework, BASEL, Basilea. (online) http://wwwbis.org.

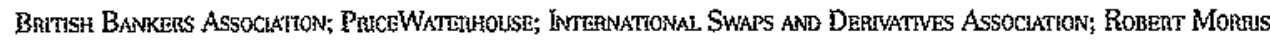
AssociaTes [1999]: Operational risk: the next frontier; (BBA, PwC, ISDA and RMA, United States).

Bello, L.; Vázquzz, R.; Trespalacios, J. A. [1993]: Investigación de mercados y estrategia de marketing, Madrid, Ed. Civitas.

CADE, E. [1999]: Managing banking risks, Chicago, Glenlake Publishing Company, Ltd.

Cica [2002]: Boletín estadistico, julio 2002, Confederacion Española de Cajas de Ahorros.

CrouHY, M.; GAiMr, D.; MARK, R. [1998]: «Operational risk and financial institutions》, Key steps in building consistent operational risk measurement and management, pp. 45-62, Arthur Andersen (Editor), Risk Books, London.

CHORAFAS, D. N. [1990]: Risk management in financial institutions, London, Butterworths.

- [2001b]: Managing operational risk. Risk reduction strategies for invesiments and commercial banks, London: Euromoney Books.

DowD, K. [2000]: Beyond value at risk, The new science of risk management, USA., Wiley.

FinANCAL, SERVCES Autionty [1999a]: Allocating regulatory capital for operational risk, FSA, United Kingdom, (online) hitp://www.fsa.gov.uk.

- [1999b]: «Senior management arrangements, systems and controls», FSA, United Kingdom, (online) htp://www:fsa.gov.uk.

- [2002]: Operational risk systems and controls, FSA, United Kingdom, (online) http:/hwwu.fsa.gov.uk.

- [2003]: Implementation of the capital accord for operational risk, ORIAG, FSA, United Kingdom, (online) http://wowfsa.gov.uk,

- [2003]: Building a framework for operational risk management: the FSA's observations, FSA, United Kingdom, (online) http://wwu.fsa.gou.uk.

Froor, K.; SCHARISTEIN, D.; STEN, J. [1994]: «A framework for risk management», Journal of Applied Corporate Finance, Vol. $7, \mathrm{n} .{ }^{\circ} 3$, pp. 22-32.

Frost, C.; Aulen, D.; Porten, J.; Bloopworti, P. [2001]: Operational risk and resilience, Understanding and minimising operational risk to secure shareholder value, (Oxford: Butterworth Heinemann).

Horram, D. G.; JorNson, M. [1996]: «Operating procedures», Risk, Vol, 9, n, ${ }^{\circ} 10$, October, pp. 60-63.

- [1998]: «Operational risk and financial institutions», New trends in operational risk measurement and management, Arthur Andersen (Editor), London, Risk Books, pp. 29-42.

- [2001]: Operational risk management: a board level issue, Bank Accounting \& Finance, Vol. 14, Issue 2, pp. 21-30. 
Homiman, D. G.; JoHNSon, M. [2002]: Managing operational risk, United States: Wiley Finance.

HuSSAIN, A [2000]: Managing operational risk in financial markets, London: Butterworth Heinemann. INTERNATIONAL SWAPS AND DERRATIVS ASsOCATION [2000b]: Operational risk regulatory approach. Discussion Paper, ISDA.

JAN WAN DEN BRINK, G. [2002]: Operational risk, The new challenge for banks (Great Britain: Palgrave). IAMESON, R. [1998]: «Derivatives and the internal auditor. Key topies and concerns», Operational risk management and measurement in financial institutions, (PwC (Editor), London, Risk Books, pp. 139-160.

KaNNTT, R. [2001\}: «Advances in operational risk. Firm-wide issues for financial institutions», How introduce an effective operational risk management framework, Andersen (Editor). London, Risk Books, pp. 67-86.

KNNEAR, T. C.; TAYLoR, J. R. [2000]: Investigación de mercados, Un enfoque aplicado, McGraw Hill, Madrid.

KNG, K. L. [2001I: Operational risk, United Kingdom: Wiley Finance.

LAM, I., y CAMmoN, G. [1998]: «Operational risk and financial institutions», Measuring and managing operational risk within an integrated risk framework (putting theory into practice), Arthur Andersen (Editor), Rísk Books, London, pp. 81-94.

Occ [2003]: Supervisory guidance on operational risk advanced measurement approaches for regulatory capital, Office of the Comptroller of Currency.

OHEY, D. [1992]: «Readings in accounting for management control», The contingency theory of management accounting achievements and prognosis, United Kingdom, Chapman \& Hall, pp. 83-106.

PriceWATERHousB [2000]: Risk management for financial institutions, Advances in measurement and control, Risk Publications, London.

- [2002]: Gestión del riesgo operacional en las entidades financieras españolas, PwC.

THE CANADIAN TREASURER [19981: The final frontier: managing operating risk, KPMG, Canada.

THE FUTURES AND OPIIONS AssoctaTION [2000a]: A review of regulatory capital requirements for EU credit institutions and investment firms. Consultation Document, FOA, United Kingdom.

- [2000b]: Operational risk, The viability of an insurance capital market solution, FOA, United Kingdom. TUFANO, P. [1996]: «Who manages risks? An empirical examination of risk management», practices in the gold mining industry》, Joumal of Finance, Vol. 51, pp. 1.097-1.137. 


\section{ANEXO}

\section{Cuestronano on Rasgo Operacionaz}

ENCUESTA SOBRE RIESGO OPERACIONAL UNIVERSDAD DE CANTABRIA

Grupo de Investigación de Contabilidad y Auditoría

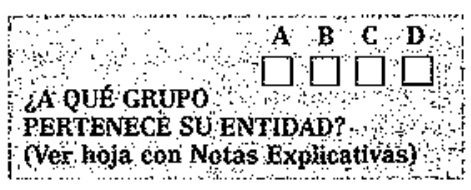

1. ¿Cómo se define en su entídrd el riesgo operacional?

2. A menudo se recurre a las subcategorias para ciarificar qué es lo que se considera como rỉesgo operacional ¿cuáles de las sigutentes estarian incluidas en su definición?

$\square$ Riesgo de crue se ejecuten incorrectamente las transacciones $\square$ Fiesgo de pérdida por la realizacion de actividades no autorizadas $\square$ Riesgo de que el personal no trabaje correctamente o cometa fallos

$\square$ Riesgo sistémico
Riesgo de errores en los registros contables

ఏ Riesgo de falio en los controles internos $\square$ Riesgo de reputación o de que se dañe la imagen $\square$ Riesgos asociados a la tecnología empleada o las aplicaciones informáticas implantadas $\square$ Riesgo de tomar decisiones inadecuada como consecuencia. de una información incorrecta

$\square$ Ritesgo de fraudes internos $o$ externos

$\square$ Ríesgo estratégico o de negocio

$\square$ Riesgo legal

○.

La definiciôn del Comité de Basilea revisada el pasado mes de julio establece que: «Piesgo operacional es el riesgo de pérdidos como resultado de procesos internos inadecuados o defectuosos, personal y sistemas o como resultado de acontecimientos externos. Seincluye el riesqo legal pero se excluye el riesgo estrotégico de reputación y sistémico"s.

3. ¿Estă de acuerdo con la definición adoptada por el Comité de Basilea en el Nuevo Acuerdo de Capital? Si no está de acuerdo, ten qué aspectos discrepa?

4. En Su organización ¿Han modificado su definición como consecuencia de las recomendaciones y avances hechos por Basilea?

$$
\text { Si } \square \quad \text { No } \square \quad \text { Aún no, pero está previste } \square
$$

5. ¿Están de acuerdo con la incorporación del riesgo operacional entre las extigencias de Capital de Basilea? 
6. ¿Qué enfệte tienen previste adoptar en su entidad para el calculo del capital económico per riesgo operacienal?

Básico $\square \quad$ Estíndar $\square \quad$ Avanzado (AMA) $\square \quad$ Aún no previsto

7. A pcsar do que el riesgo oporacional no sea algo nuevo para las entidndes, se hak Rpreciudo un creciente interés por éi en los flitimos años, za qué cree usted que se ha debido?

Volore en lina escalia de 1 a $5: \quad 1=$ poco importante, 5 = muy importanto)

प. A las exigenżas de capital por riesgo operaclonal según el Nuevo Acuordo do Capital de Basilea.

$\square$ Al aumento percibido de esto riesgo como consecuenpia de la globalizacion, creciente complejidad do instrumentos, nuevos mercados, $\theta$-business, etc.

$\square$ A las importantes pérdidas sufridas internamente en su entidad atribulbles a este riesgo.

$\square$ A las importantes pérdidas sufrtdas por otras entidades atributbies a este riesgo.

$\square$ Al interés por controlar y reducir pordidas oporacionales.

$\square$ A la bísqueda de ventajas competitivas.

$\square$ A la nueva tendencia de integrar el riesgo en las entidades, como consecuencia légica después de abordar el riesgo de mercado y el de crécito.

$\square$ A la creciente atención de los reguladores sobre este riesge.

$\square$ A la concienciación de la alta direceion con este rtosgo.

Q Otros ¿Cuáles?

8. Como consecuencia de teilo ello, dhan adoptado alguna medida en su ontidad?
SI
No
No, pero está previsto

Si su respuesta es afîmativa ¿desdio g̨ué año?

¿Cuáles?

9. ¿Existe un área indepondiente de gestión de riesgo operacional? Si $\square$ No $\square$

10. ¿Existe un Comité de riesgo operacional? Si $\square$ No $\square$ Está en desarrollo

11. ¿Existo una Base de Datos de pórdida opcracionales? Sí $\square$ No $\square$ Está en desarrollo $\square$ Si su respuesta es afirmativa ¿desde qué as̃o??

12. ¿Cuăl es eì papel de la Auditoria Interna frente al riesge operaciongl?
Supervisión
Seguimiento
Proveedor de información
Responsable principal
del riesgo operacional
Lidera las operaciones $\square$ de gestion del riesgo

13. ¿Quién se ocupa de la gestión, control y supervisión del riesgo operacional en su entiład?

\begin{tabular}{|c|c|c|c|c|c|c|c|}
\hline & $\begin{array}{c}\text { Bpto. de } \\
\text { Gestión de } \\
\text { Riesgos }\end{array}$ & $\begin{array}{c}\text { Dpto. de } \\
\text { Auditoría } \\
\text { Interna. }\end{array}$ & $\begin{array}{c}\text { Dpto. de } \\
\text { Riesgo } \\
\text { Operacional }\end{array}$ & $\begin{array}{c}\text { Cada ma de } \\
\text { las unidades } \\
\text { de negocio }\end{array}$ & $\begin{array}{c}\text { De forma } \\
\text { específica } \\
\text { nadie }\end{array}$ & $\begin{array}{l}\text { Aûn no está } \\
\text { previsto }\end{array}$ & 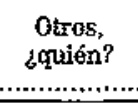 \\
\hline Gestúon & $\square$ & $\square$ & $\square$ & $\square$ & $\square$ & $\square$ & $\square$ \\
\hline Control & $\square$ & $\square$ & $\square$ & $\square$ & $\square$ & $\square$ & $\square$ \\
\hline Supervisión & $\square$ & $\square$ & $\square$ & $\square$ & $\square$ & $\square$ & $\square$ \\
\hline
\end{tabular}

14. ¿Cree que su entidad estará preparada paxa aplicar los métodes propuestos por Basilea para el 2007 ? Si $\square$ No

15. De 1 a 10 come valoraría la preparación e dessarrollo de su entifad en este tema 